\title{
Special deceleration strip design of Yaxi Expressway
}

\author{
Qing Chen ${ }^{1}$, Sixia Wang ${ }^{1}$ \\ ${ }^{1}$ Sichuan Vocational and Technical College of Communications, P.O. Box: Wenjiang District, Liu \\ TaiRoad, No. 208 City: Chengdu, Sichuan, 611130
}

scjychenqing@126.com

\begin{abstract}
Keywords: Highway; Traffic Engineering of communication; Mountainous Highway Long Downhill; Deceleration strip.

Abstract. We elaborate the special deceleration strip design ideas of Yaxi Expressway; put forward the special deceleration strip design plan of Yaxi Expressway. We draw a conclusion that the special deceleration strip of Yaxi Expressway can effectively restrain highway major traffic accidents in the long downhill sections of the mountainous area through the car and wagon ride comfort test on the special deceleration strip.
\end{abstract}

\section{Introduction}

Yaxi Expressway is located in the connecting area of Qinghai-Tibet Plateau and Sichuan basin. Its geological condition is very complicated; it climbs 6 mountains, forms 3 long continuous longitudinal slopes- in the whole line. The continuous line of vertical slope length of the North slope, South Slope of Daxiangling, the north slope of Tuowushan is respectively $33 \mathrm{~km}, 26 \mathrm{~km}$ and $51 \mathrm{~km}$. It's the longest in the country. Its average longitudinal slope is respectively $2.28 \%, 2.57 \%$ and $2.97 \%$. In the three long longitudinal slope section $22 \mathrm{~km}$ is above the snow line, and some sections at a certain altitude is also affected by ice, snow, rain, fog and other inclement weather conditions. The operation security situation is very severe, therefore, it is necessary for the downhill wagon to adopt a more scientific management measures.

\section{The necessity of using deceleration zone in expressway and the design ideas}

The special expressway deceleration strip is based on the severe expressway traffic safety situation emerges as the times require an enhanced road safety facilities. According to the vehicle speed, traffic structure, the actual average speed, accident characteristics, road alignment, pavement materials and other specific conditions, we design deceleration strip shape and size (width, height), coupling installation, material, processing technology and other factors; when necessary theoretical calculation and analysis test must be taken. Its ultimately target is safe use, without damaging the road surface, effectively to control the vehicle speed.

The deceleration strip of expressway, unlike the current district, city roads and toll station entrance uses hump type deceleration zone, overcomes its vehicle vibration generated by higher speed, which easy to cause the discomfort and damaged the car parts, ensures the car safety at high speed and the larger car needs a downshift speed.

\section{Necessities}

At present highway construction is pushing forward to the mountainous area .The high mountains, steep slopes, narrow curved roads and crosses, natural disasters, low vehicle operation safety condition is new challenges to expressway constructions in western areas. Our automobile technology is relatively backward and the quality of truck driver is low. Relying on the traditional security facilities (such as: the speed limit signs, mandatory parking area etc) cannot contain the new safety problems of long downhill sections of Expressway in mountainous area.

High grade highway special deceleration strip put into use as early as 2003 in long downhill sections (K1562-K1569) of Shaanxi G312 Xianyong I class highway, and later in Yunnan Songdai Highway 
Long Downhill sections and Yunnan Luofu expressway known as the "road of death". Its containment effect is very obvious to freight traffic accident caused by long downhill braking failure malignant.

Therefore using conventional passive safety measures can not ensure the safety, developing and using a special deceleration appropriate for Yaxi Expressway to actively control speed, avoid serious traffic accidents is worth learning in the Yaxi expressway.

\section{Design ideas}

\section{(1)Active compulsive speed control}

The principle of expressway special deceleration strip is actively arranging specially designed deceleration zone in special sections (sharp or continuous long downgrade and accident-prone sections). When the speed is high it will produce intense mechanical vibration, the vibration from the tire transports to the driver through the body and seat, causes him feeling very uncomfortable. In addition, according to the common sense the driver will estimate it should bring severe vibration of the vehicle, may cause damage to the vehicle and the goods when through the deceleration zone. The physiological and psychological feeling of foresight can reduce the traffic safety of the driver, so that the vehicle driver will consciously take the initiative to reduce speed to improve safe driving in the special sections.

\section{(2) Comply with systematic principle}

The installation location and quantity of expressway special deceleration strip which is to improve long downhill sections driving can not be determined casually, must be systematic. The traffic flow characteristics, potential unsafe factors, accident characteristics and road conditions must be considered. It should be determined after detailed investigation and analysis of system to achieve the effective organization and control of compulsive speed control objective. Otherwise, it will decrease the effect, even bring negative effect.

In order to overcome the long downhill in Yaxi Expressway, we have considered the highway alignment consistency, balance and people (the driver) - car - road joint operation coordination. According to the expressway construction design parameters of different models, we calculated the specific downhill predicting speed, brake thermal efficiency of recession (the long downhill braking speed control safety performance). According to the results of calculation, we brought out the corresponding solution, installing specific special deceleration strip in different downhills, so that the truck driver will decrease driving speed, reaching the safety objective.

(3) Ensuring the high speed, safety

At present, most of the cars use independent suspension. Its stiffness is little, the vibration frequency is low, and smoothness is good. The vibration generated when the vehicle drives across the road bring little impact to the passengers. The large cars use suspension with longitudinal leaf spring as the elastic element, its stiffness is high, and vehicle driving conformance is poor, vibration generated when the vehicle drives across the uneven road when the driver has a strong discomfort. According to car and large car suspension performance differences, we designed a special deceleration strip with a specific section width and height, which are suitable for the characteristics of expressway.

After the installation of special deceleration strip it can be ensured that cars with higher speed can pass without too big impact to ensure the high speed fluid characteristics; and large cars through the deceleration band will produce a stronger sense of discomfort, forcing the driver to control the speed of the vehicle low, thereby avoiding braking failure caused by overheating at a high speed, so that improving the long downhill driving safety.

\section{Design plan}

\section{Structure design of the deceleration strip}

According to the primary design of speed standard, combining the safety belt use research results of Yunnan Songdai highway and Shaanxi Xianyong I highway, we comprehensive considered the ride comfort of the connecting point between the wheel and the deceleration point and vibration effect, then 
decided the deceleration zone geometry: long, wide, section height are $700 \mathrm{~mm}, 500 \mathrm{~mm}, 35 \mathrm{~mm}$, the angle between ground and hypotenuse is 14 degrees. At the same time, in order to improve the service life of the deceleration strip, the internal structure is wire mesh and sheet steel which increase its tensile strength. It use wedge connecting structure between adjacent deceleration block, reinforced by bar connecting structure, improves the integral force performance, prolongs the service life. Design of the structure size of the deceleration strip is shown as in Figure1, the deceleration strip is arrangement shown as in figure2.

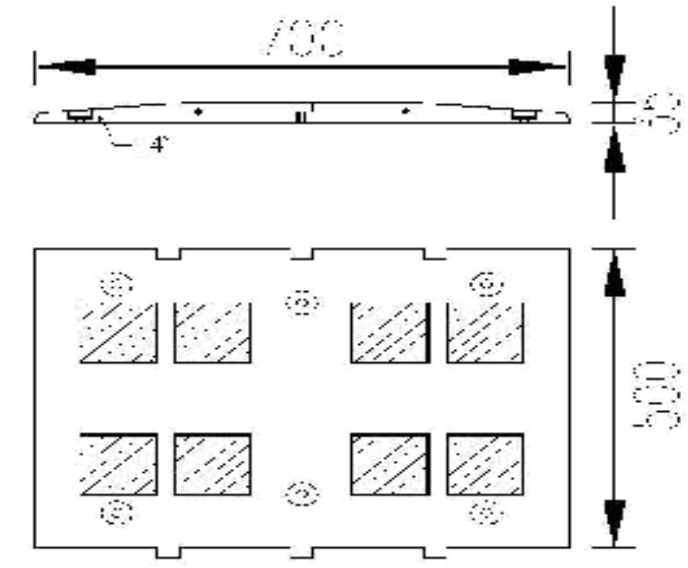

fig1 size of the deceleration strip

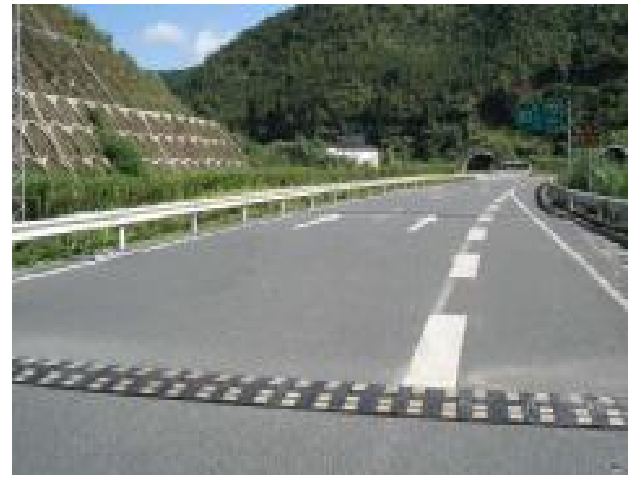

fig2 deceleration strip arrangement

\section{Deceleration strip arrangement design}

\section{(1)The north slope of Tuowu Mountain}

The north slope of Tuowu Mountain is up to $51 \mathrm{~km}$. The Liziping, Ganhaizi forced parking area, the Pushagang service area and the two small radius spiral tunnel Ganhaizi and Tiezaizi are key safety monitoring points. Based on the actual situation of section, we put forward the following proposals to control downhill speed for truck safety:

(1) Set 1 group of special deceleration strip nearby Liziping forced parking area, K142+900 (left), to force the bus, truck decrease speed and get into the parking area.

(2) Set 1 group of special deceleration strip nearby Ganhaizi spiral tunnel, K151+900 (left), to force the vehicle actively decrease speed before entering the tunnel.

(3) Set 1 group of special deceleration strip nearby Ganhaizi forced parking area, K154+500 (left), to force the vehicle decrease speed and get into the parking area.

Set 1 group of special deceleration strip nearby Ganhaizi spiral tunnel in a right location , K142+900 (left), to force the vehicle actively decrease speed before entering the tunnel.

(4) Set 1 group of special deceleration strip nearby Pushagang parking area, K142+900 (left), to force the bus, truck enter the parking area to check the vehicle condition to prepare downhill.

Set 1 group of special deceleration strip nearby Yihai safety inspection station, K191+400 (left), to force the large trucks decrease speed actively into the checkpoint to be inspected. Forbid "super three" and dangerous goods vehicle driving in the longitudinal slope section.

(2) The northern slope of Daxiangling (K53+250 - K26+350)

Set 1 group of special deceleration strip on the northern slope of the outlet, K53+700 (left), to force vehicles decelerate actively, prevent too high speed and out of controlling on the winter ice road.

Because Yingjing service area is located on the northern slope of the bottom of Daxiangling, the speed of vehicles is high before entering the service area, we recommend set 1 group of special deceleration strip before the service area, pile number K28+300 (near the left line), to force the large vehicle decelerates actively before entering the service area, then safely enters the service area.

Set 1 group of special deceleration strip nearby Yingjing safety inspection station, K24+200 right), to force the large trucks decrease speed actively into the checkpoint to be inspected.

(3)the south slope of Daxiangling (K64+050 - K64+720) 
Set 1 group of special deceleration strip on the northern slope of the outlet, K63+850 (right), to force vehicles decelerate actively, prevent too high speed and out of controlling on the winter ice road.

\section{Automotive ride comfort test for special deceleration strip}

The research has shown in order to maintain a good vehicle ride comfort, the vehicle body natural vibration frequency should be accordance with the human body walking, exercise frequency, is about $1 \mathrm{HZ} 1.6 \mathrm{HZ}$, vibration acceleration maximum value is $0.3 \sim 0.4 \mathrm{~g}$. In order to ensure the goods integrity, body vibration acceleration also should not be too big, if the vehicle body acceleration is up to $1.0 \mathrm{~g}$, the goods without fixed is likely to leave the compartment floor. Therefore, the body vibration acceleration maximum value should be less than $1.0 \mathrm{~g}$.

\section{Vibration acceleration detection on the deceleration zone}

After Luofu expressway deceleration zone installation, we selected cars and three axis trucks as the test vehicles. The car through the deceleration strip test is shown as Figure 3, with the 32 channel M3INTE6RR2 signal acquisition instrument to do the ride comfort test. When test mounting vibration sensors respectively in the car front seat, before the floor, respectively test the vibration acceleration on each test point in a vertical direction o at 40,50,60,70,80km/h per hour. The test results are shown as figure 4- shown in figure 5:

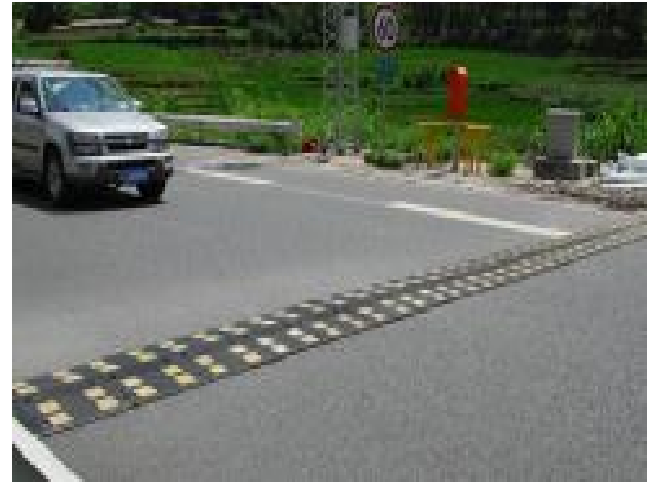

Fig3 vibration acceleration detection on the deceleration zone

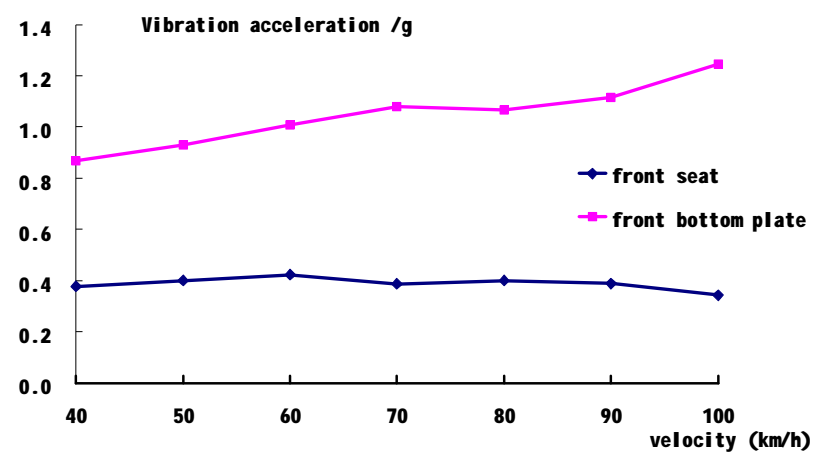

Fig4 Vibration acceleration of the front seat and the front bottom plate

Figure 4 shows: when test vehicle at different speeds drove on the deceleration zone, because the front independent suspension and the double damping effect of the seats, the vertical vibration acceleration of the front seat maintained at around $0.4 \mathrm{~g}$. The vertical vibration acceleration of the bottom plate (body) at $60 \mathrm{~km} / \mathrm{h}$ boundaries: speed below $60 \mathrm{~km} / \mathrm{h}$, acceleration was less than $1.0 \mathrm{~g}$, smaller than the maximum value; when the speed was higher than $60 \mathrm{~km} / \mathrm{h}$, acceleration is bigger than $1 \mathrm{~g}$, exceeded the maximum value, although did not cause too big change of the ride comfort, made too shock and vibration, too big noise.

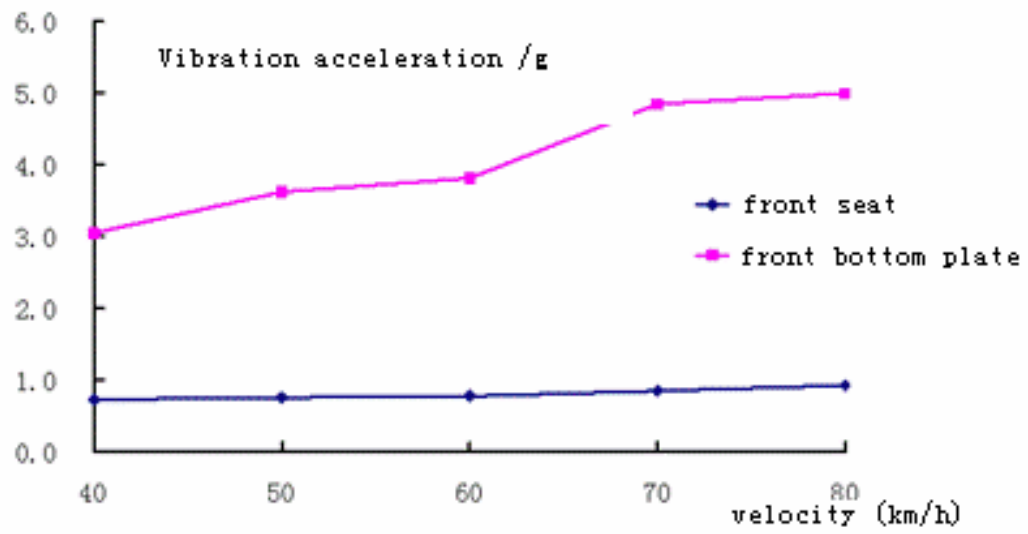


Fig5 Vibration acceleration of back the seat and the compartment

Figure 5 shows: when the test vehicle at $40-80 \mathrm{~km} / \mathrm{h}$ speed went through the deceleration zone, as the truck suspension elastic element was plate spring, damping effect was poor, with the double damping effect of the seat; the vertical vibration acceleration of the seat was between 0.8 and $1.0 \mathrm{~g}$, and with the speed increased. Therefore, the vertical vibration acceleration of the seat has reached the limit value; the ride comfort is poor. At the same time, before the vertical vibration acceleration of the bottom got up more than $3 \mathrm{~g}$, the higher the speed, the stronger the vibration was.

\section{Subjective evaluation of comfort}

For subjective evaluation, we chose 2 drivers, 4 passengers compose the test group. They respectively took cars and 3 axle wagons $(6 \times 4)$ at $30,40,50,60,70,80 \mathrm{~km} / \mathrm{h}$ speed on the deceleration zone, feeling the ride comfort. The three axle truck on the deceleration zone was shown in figure 6 .

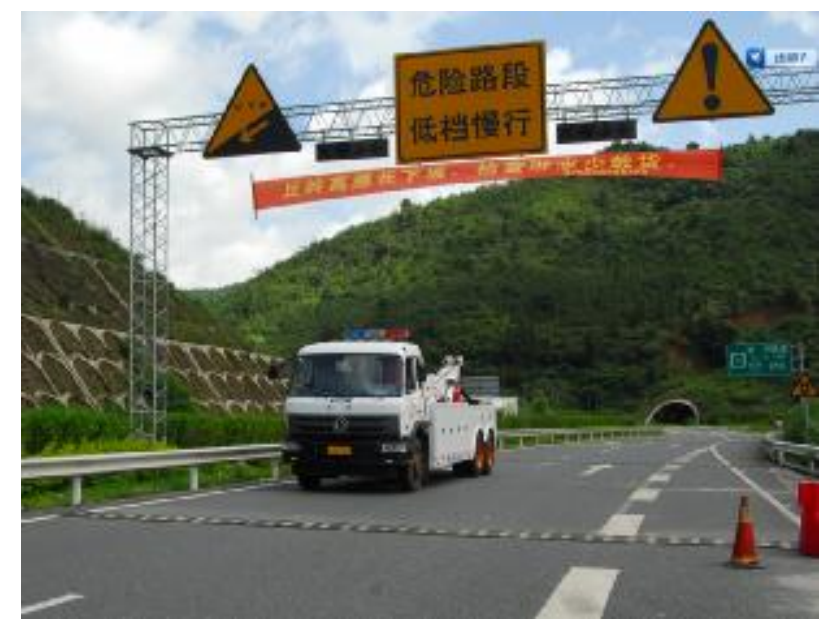

Fig 6 three axle truck test

Test results show that: when the car was below $60 \mathrm{~km} / \mathrm{h}$ through the deceleration zone, the driver and passengers can accept vibration, above $60 \mathrm{~km} / \mathrm{h}$ through the deceleration zone, the driver and the passengers felt discomfort reaction strong, the driver controlled the direction difficultly; when the truck was below $40 \mathrm{~km} / \mathrm{h}$ speed through the deceleration zone, the driver and passengers can accept vibration, the compartment amplitude is small, more than $40 \mathrm{~km} / \mathrm{h}$ through the deceleration zone, the driver and passengers felt strong vibration, compartment bumped large noise, the driver would initiative downshift.

\section{Conclusion}

Referring the long downhill deceleration strip's successful experience from G312 Shaanxi Xianyong I class highway, Yunnan Songdai highway and Yunnan Luofu highway, combined with the characteristics of Yaxi Expressway special deceleration strip design, contrasted the cars and trucks ride comfort test, Yaxi special long downhill deceleration strip can make the car at $60 \mathrm{~km} / \mathrm{h}$ quickly through, and truck must be at $40 \mathrm{~km} / \mathrm{h}$ through. It has obvious effect on improving the running safety of the vehicle and was worth using for reference in the western mountainous area in the high grade highway long down slope section in our country.

\section{References:}

[1] Xiao runmou, Wang Jianbo, Zhou Weixin. Special deceleration strip design and application for Long downgrade section of Expressway in mountain area [J]. highways \& automotive applications. 2010.03

[2] Chen Bin, Yuan Wei, Fu Rui, Guo yingshi. Continuous long downgrade section feature analysis of the traffic accidents [J]. Journal of traffic and transportation engineering..2009.9 (4). 
[3] Western traffic construction project of science and technology. Continuous long downgrade section Security Technology Research Report.2007.

[4] Safety engineering center of Ministry of traffic. Badaling highway vehicle continuous downhill test report.2003

[5] Kay Fitzpatrick.Lily Elefteradou et al.Speed Prediction for Two-Lane Rural Highways.Publication NO.99-171.Auguest 2010,100-106

[6]Douglas W.Harwood etc.Commercial Truck and Bus Safety Stnthesis 3:Hiaghway/Heavy Vehicle Interaction[R].TRB,2009 